\title{
Adeno-associated virus-8-Mediated intravenous transfer of myostatin propeptide leads to systemic functional improvements of slow but not fast muscle
}

Article

Published Version

Foster, K., Graham, I. R., Otto, A., Foster, H., Trollet, C., Yaworsky, P. J., Walsh, F. S., Bickham, D., Curtin, N. A., Kawar, S. L., Patel, K. and Dickson, G. (2009) Adenoassociated virus-8-Mediated intravenous transfer of myostatin propeptide leads to systemic functional improvements of slow but not fast muscle. Rejuvenation Research, 12 (2). pp. 85-93. ISSN 1549-1684 doi: https://doi.org/10.1089/rej.2008.0815 Available at https://centaur.reading.ac.uk/29504/

It is advisable to refer to the publisher's version if you intend to cite from the work. See Guidance on citing.

Published version at: http://online.liebertpub.com/doi/abs/10.1089/rej.2008.0815

To link to this article DOI: http://dx.doi.org/10.1089/rej.2008.0815

Publisher: Mary Ann Liebert Inc.

All outputs in CentAUR are protected by Intellectual Property Rights law, including copyright law. Copyright and IPR is retained by the creators or other copyright holders. Terms and conditions for use of this material are defined in the End User Agreement. 


\section{www.reading.ac.uk/centaur}

\section{CentAUR}

Central Archive at the University of Reading

Reading's research outputs online 


\title{
Adeno-Associated Virus-8-Mediated Intravenous Transfer of Myostatin Propeptide Leads to Systemic Functional Improvements of Slow but Not Fast Muscle
}

\author{
Keith Foster, ${ }^{1}$ Ian R. Graham, ${ }^{1}$ Anthony Otto, ${ }^{2}$ Helen Foster, ${ }^{1}$ Capucine Trollet, ${ }^{1}$ Paul J. Yaworsky, ${ }^{3}$ \\ Frank S. Walsh, ${ }^{3}$ Dale Bickham, ${ }^{4}$ Nancy A. Curtin, ${ }^{4}$ Susannah L. Kawar, ${ }^{1}$ Ketan Patel, ${ }^{2}$ \\ and George Dickson ${ }^{1}$
}

\begin{abstract}

\section{Introduction}

$\mathbf{T}$

HE LOSS OF SKELETAL MUSCLE TISSUE has a major impact on public health. A variety of conditions result in the loss of muscle, including disease-related loss (cachexia), age-associated loss (sarcopenia), enforced inactivity, such as bed rest and muscular dystrophies. ${ }^{1}$ Skeletal muscle development is a complex process, with a number of regulatory factors for the different steps involved in muscle stem cell activation, proliferation, and postmitotic differentiation having been identified. ${ }^{2}$ Skeletal muscle size is dynamic and responsive to extracellular signals such as mechanical load, neural activity, hormones, growth factors, and cytokines. However, myostatin is a central determinant of muscle size and mass; its role has been established from the effects of gene-inactivating mutations in mouse. ${ }^{3,4}$ Inactivation mutations of myostatin have now been described in several mammals such as cattle, sheep, dogs, and humans, ${ }^{5-9}$ with the central tenet being hypermuscularity and reduced adipogenesis. Conversely, the overexpression of myostatin is a key feature in cachexia ${ }^{10,11}$ and sarcopenia. ${ }^{12}$
\end{abstract}

Myostatin is a member of the transformating growth factor- $\beta$ (TGF- $\beta$ ) superfamily of proteins and is produced almost exclusively in skeletal muscle tissue, where it is secreted and circulates as a serum protein. Myostatin acts as a negative regulator of muscle mass through the canonical SMAD2/3/4 signaling pathway. Naturally occurring myostatin mutants exhibit a 'double muscling' phenotype in which muscle mass is dramatically increased as a result of both hypertrophy and hyperplasia. Myostatin is naturally inhibited by its own propeptide; therefore, we assessed the impact of adeno-associated virus-8 (AAV8) myostatin propeptide vectors when systemically introduced in MF-1 mice. We noted a significant systemic increase in muscle mass in both slow and fast muscle phenotypes, with no evidence of hyperplasia; however, the nuclei-to- cytoplasm ratio in all myofiber types was significantly reduced. An increase in muscle mass in slow (soleus) muscle led to an increase in force output; however, an increase in fast (extensor digitorum longus [EDL]) muscle mass did not increase force output. These results suggest that the use of gene therapeutic regimens of myostatin inhibition for age-related or disease-related muscle loss may have muscle-specific effects.

Myostatin is a member of the transforming growth factor$\beta$ (TGF- $\beta$ ) superfamily and is predominantly expressed in and secreted from skeletal muscle. It is expressed throughout embryonic and fetal development and in adult muscle, which suggests a role in both prenatal muscle development and in maintenance and/or repair of postmitotic muscle. ${ }^{3,13}$ Like other TGF- $\beta$ family members, it is first synthesized as a precursor protein (376 amino acids) that is processed at a conserved cleavage site by calcium-dependent furin-like proteases to yield the amino-terminal propeptide (or latency-associated peptide) and the carboxy-terminal mature peptide. ${ }^{4}$ The amino-terminal propeptide is capable of maintaining the carboxy-terminal dimer in a latent state both in vitro and in vivo, thus inhibiting its biological activity. ${ }^{4,14-16}$ This is also achieved when circulating myostatin interacts with proteins such as follistatin, follistatin-related gene, and GDF-associated serum protein-1 (GASP-1). ${ }^{4,14,17}$

It is becoming increasingly clear that the role of myostatin in determining myogenic cell proliferation and differentiation is highly complex. In the canonical signal transduction

${ }^{1}$ SWAN Institute of Biomedical and Life Sciences, School of Biological Sciences, Royal Holloway-University of London, Egham, UK. ${ }^{2}$ School of Biological Sciences, University of Reading, Reading, UK.

${ }^{3}$ Clinical Discovery, Wyeth Research, Collegeville, Pennsylvania.

${ }^{4}$ National Heart and Lung Institute, Imperial College London, London, UK. 
pathway, the propeptide domain is activated by the bone morphogenetic protein-1 (BMP-1)/tolloid family of metalloproteases to release the mature peptide. ${ }^{18}$ The 'free' mature peptide complexes the cell-surface activin receptor type $\mathrm{IIb}$, which recruits and phosphorylates activin receptor type I. Subsequently, these heteromeric activin receptors phosphorylate SMAD2/3, and then bind co-SMAD4, facilitating translocation to the nucleus; once there, they act as a transcriptional modulator, affecting both muscle cell proliferation and differentiation. ${ }^{19-22}$

Myostatin has recently been shown to affect signal transduction by both SMAD3-independent and -dependent pathways. First, myostatin has been shown to abrogate the mitogenic effects of the insulin-like growth factor- $1 /$ phosphoinositide-3 kinase/Akt (IGF-1/PI3K/Akt) nexus by targeting cyclin D1 for polyubiquitination, resulting in $\mathrm{G}_{1}-\mathrm{S}$ progression failure. ${ }^{23,24}$ Another sequela of perturbing the IGF-1/PI3K/AKT pathway is that myostatin induces hypophosphorylation of AKT, resulting in diminished phosphorylation levels of the transcription factor FoxO1, thus increasing the levels of atrogin-1, a potent activator of the ubiquitin E3 ligase. Additionally, myostatin can activate the TGF- $\beta$-activated kinase 1 (TAK- 1 ), leading to activation of the p38 mitogen-activated protein kinase (MAPK) and upregulation of the cyclin-dependent kinase inhibitor P21, affecting the phosphorylation status of the retinoblastoma protein. ${ }^{20,25,26}$ These processes are independent of SMAD3. However, importantly, SMAD3-dependent interactions induced by myostatin affect the Wnt signaling cascade, either by formation of smad3/b-catenin/TCF-4 complex ${ }^{27}$ or interacting with other Wnt signaling intermediates. ${ }^{28}$

Whereas myostatin has been described as the muscle chalone, it is becoming increasingly apparent that it is not the sole mediator of muscle mass. It has been shown that the interference of other TGF- $\beta$ family members coordinates signals to manage the activation and differentiation of muscle. ${ }^{29,30}$

Given that myostatin is naturally inhibited by its own propeptide, we have generated adeno-associated virus- 8 (AAV8) vectors expressing a mutagenized propeptide fused to a mouse immunoglobulin Fc molecule. ${ }^{18}$ AAV8, while able to transduce muscle, is a potent transducer of liver tissue, therefore ensuring ectopic production of the transgene from the liver into the serum. ${ }^{31}$ We have used this vector to assess the histological and physiological properties of muscle following systemic administration in normal muscle to evaluate its potential use in muscle-wasting diseases and disorders.

\section{Materials and Methods}

\section{Generation of vectors}

The myostatin propeptide cDNA was obtained from Paul Yaworsky (Wyeth Research, USA); the myostatin propeptide sequence had been previously modified and fused to mouse immunoglobulin $\mathrm{G}_{2 \mathrm{a}}\left(\operatorname{IgG}_{2 \mathrm{a}}\right)$ moiety. ${ }^{18}$ The cDNA sequences were cloned into a pDD-derived AAV plasmid under the control of the CAGGs promoter ${ }^{32}$ to generate the plasmid pProMyo. AAV8ProMyo vectors were produced and viral genome titered by Nantes Vector Core (France).

\section{Administration of $A A V$}

Outbred MF-1 male mice were housed in minimal disease facilities (Royal Holloway, University of London) with food and water ad libitum. At 6 weeks of age, the mice were maintained at $40^{\circ} \mathrm{C}$ for $10 \mathrm{~min}$ housed in an incubator (Datesand Ltd, Manchester, UK) prior to a viral injection. Mice were restrained and injected via the tail vein with $5 \times 10^{11}$ vector genomes. Histological assessments of tibialis anterior (TA) and gastrocnemius muscles were performed at 4 weeks and 10 weeks following administration of AAV. Physiological assessment of soleus and extensor digitorum longus (EDL) muscle was performed at 4 weeks postadministration of AAV. Tail vein bleeds of $75 \mathrm{uL}$ were taken at 1, 4, and 10 weeks postadministration of AAV, and transgene expression was assessed by western blotting.

\section{Detection of transgene expression from serum samples}

Plasma samples were pooled and diluted 1 in 150 with $1 \times$ phosphate-buffered saline (PBS). A total of $5 \mathrm{uL}$ was denatured by the addition of sodium dodecyl sulfate-polyacrylamide gel electrophoresis (SDS-PAGE) sample buffer containing $5 \mathrm{mM}$ (wt/vol) dichloro-diphenyl-trichloroethane and heating at $100^{\circ} \mathrm{C}$ for $5 \mathrm{~min}$. Samples were then subjected to $4-12 \%$ SDS-NuPAGE electrophoresis (Invitrogen, Paisley, UK), and resolved proteins were transferred to Hybond-ECL membranes (GE Healthcare, Amersham, UK).

Nitrocellulose blots were incubated with goat anti-mouse $\mathrm{IgG}_{2 \mathrm{a}}$ antibody (1:50 dilution; $\mathrm{AbD}$ Serotec, $\mathrm{UK}$ ) to detect the $\mathrm{IgG}_{2 \mathrm{a}}$ moiety of the ProMyo-IgG $\mathrm{I}_{2 \mathrm{a}}$ Fc fusion transgene product, followed by an anti-goat-horseradish peroxidase (HRP) secondary antibody (1:3000 dilution; Sigma-Aldrich, Poole, UK). Blots were developed using the ECL detection system (GE Healthcare). Samples were compared against conditioned media recovered from HEK293T cells transfected with pProMyo (positive control) and serum derived from uninjected MF-1 males. No endogenous myostatin is detected by this method.

\section{Histology}

Recovered tissues were mounted in Cryo-M-Bed (Bright Instruments, Huntingdon, UK) and snap-frozen in liquid nitrogen-cooled isopentane. Laminin- $\alpha 2$ staining was carried out on 10-um cryosections. Frozen tissue sections from control and treated mice were air dried for $30 \mathrm{~min}$ and blocked in 5\% marvel (wt/vol) in PBS/0.05\% Tween 20 . Laminin- $\alpha 2$ was detected with a primary antibody (1:100 dilution; SigmaAldrich) followed by an anti-rat-HRP secondary antibody (1:3000 dilution; Sigma-Aldrich).

For muscle fiber typing, the primary antibodies used were monoclonal mouse anti-type I myosin heavy chain (MHC) IgM (Developmental Studies Hybridoma Bank, IA) at a dilution of 1:1, monoclonal mouse anti-type IIa MHC IgG (Developmental Studies Hybridoma Bank, IA) at a dilution of 1:4 and monoclonal mouse anti-type IIb MHC IgM (Developmental Studies Hybridoma Bank, IA) at a dilution of 1:1. MHC type I and type IIB primary antibodies were detected using Alexa Fluor 633 fluorochrome-conjugated goat antimouse IgM (Invitrogen; 1:200); mouse primary antibody MHC type IIa was detected using Alexa Fluor 488 fluorochrome-conjugated goat anti-mouse IgG (Invitrogen; 1:200). Samples were incubated in primary antibodies for 18 $\mathrm{h}$ at $4^{\circ} \mathrm{C}$, the anti-rabbit secondary antibody was incubated for $1 \mathrm{~h}$ at room temperature and all fluorescent antibody labeling was carried out at room temperature for $45 \mathrm{~min}$. Subsequent image analysis was performed using a Zeiss Axio- 
scop2 fluorescent microscope, an axiocam digital camera, and Zeiss axiovision software.

\section{Single myofiber isolation, satellite cell, and nuclei counts}

Individual myofibers were isolated from the EDL muscle and cultured as described previously. ${ }^{33}$ Briefly, EDL muscles were digested in $0.1 \%(\mathrm{wt} / \mathrm{vol})$ type 1 collagenase in Dulbecco modified Eagle medium (DMEM) containing Glutamax and $100 \mu \mathrm{g} / \mathrm{mL}$ penicillin/streptomycin for $2 \mathrm{~h}$ at $37^{\circ} \mathrm{C}$. Using glass pipettes, single myofibers were separated from the muscle bulk, washed in DMEM, and either fixed immediately in $2 \%$ paraformaldehyde in PBS for $10 \mathrm{~min}$ or cultured. Myofibers were mounted using a fluorescent mounting medium (Dako) containing $2.5 \mu \mathrm{g} / \mathrm{mL} \mathrm{4',6-di-}$ amidino-2-phenylindole (DAPI) for nuclear visualization. Fixed myofibers were permeabilized in a solution of $20 \mathrm{mM}$ HEPES, $300 \mathrm{mM}$ sucrose, $50 \mathrm{mM} \mathrm{NaCl}, 3 \mathrm{mM} \mathrm{MgCl}_{2}$, and $0.5 \%$ Triton $\mathrm{X}-100, \mathrm{pH} 7$, at $4^{\circ} \mathrm{C}$ for $15 \mathrm{~min}$. Fibers were washed in PBS, and nonspecific binding was blocked using wash buffer (5\% vol/vol fetal calf serum [FCS] in PBS with $0.05 \% \mathrm{vol} / \mathrm{vol}$ Triton X-100) for $30 \mathrm{~min}$. A primary antibody used to detect satellite cells was monoclonal mouse anti-Pax7 IgG (Developmental Studies Hybridoma Bank, 1:4) and was detected using Alexa Fluor 488 fluorochrome-conjugated goat anti-mouse IgG (Molecular Probes A11029, 1:200). Myofibers were mounted using fluorescent mounting medium (Dako Cytomation) containing $2.5 \mu \mathrm{g} / \mathrm{mL}$ DAPI for nuclear visualization. Subsequent image analysis and manual quantification of labeled cells was performed using a Zeiss Axioscop2 fluorescent microscope, Axiocam digital camera, and Zeiss Axiovision software version 4.7.

\section{Muscle contractile function}

Experiments were performed on soleus and EDL muscles from adult males. Mice were killed according to Schedule 1 of the Animals (Scientific Procedures) Act, United Kingdom. During dissection and experiments, muscles were bathed in a saline solution containing $118 \mathrm{mM} \mathrm{NaCl}, 4.75 \mathrm{mM} \mathrm{KCl}, 1.18$ $\mathrm{mM} \mathrm{MgSO}_{4}, 24.8 \mathrm{mM} \mathrm{NaHCO}_{3}, 1.18 \mathrm{mM} \mathrm{KH}_{2} \mathrm{PO}_{4}, 2.54 \mathrm{mM}$ $\mathrm{CaCl}_{2}$, and $10 \mathrm{mM}$ glucose, and continuously bubbled with $95 \% \mathrm{O}_{2}$ and $5 \% \mathrm{CO}_{2}$. T-shaped aluminium foil clips were folded and glued (Loctite Gel, Loctite) onto each tendon. The muscle was mounted in a Perspex bath between a combined motor and force transducer (Cambridge Technology, Inc., model 300B) and a fixed hook. Saline solution was continuously circulated through the bath and its temperature was maintained at $20^{\circ} \mathrm{C}$. Muscles were electrically stimulated (Digitimer, MultiStim System-D330) using platinum wire electrodes placed adjacent to the preparation. A program was written in TestPoint (Keithley Instruments, UK) controlled stimulation and motor arm position and recorded force, length, and stimulation. A DAS-1800AO Series A/D board (Keithley Instruments, UK) was used to send and acquire data at $1 \mathrm{kHz}$.

The relationship between the stimulus strength (V) during a single stimulus (1.0 msec pulse duration) and isometric twitch force was investigated in each muscle to establish the supramaximal stimulus strength. The relationship between length and force was investigated in each muscle to identify the fiber length $\left(L_{0}\right)$ at which the tetanic force was maximal (100 msec tetanus at approximately $75 \mathrm{~Hz}$ for soleus and $125 \mathrm{~Hz}$ for EDL). A "standard" 0.5-sec isometric tetanus at $L_{0}$ was recorded for each muscle.

At the completion of the experiment, muscles were placed in $2 \%$ paraformaldehyde overnight and then rinsed with water. Muscle length was measured under a dissecting microscope. After removing all tendon and connective tissue, the muscle fibers were dried at room temperature and weighed on a Cahn microbalance. The cross-sectional area (CSA) was calculated as:

$$
\mathrm{CSA}=(4.9 M / d) / L_{0},
$$

where $M$ is dry mass (in $\mathrm{mg}$ ), 4.9 is the wet-to-dry mass ratio, ${ }^{34} d$ is density (assumed to be $1 \mathrm{mg} \cdot \mathrm{mm}^{-3}$ ), and $L_{0}$ is muscle length (in $\mathrm{mm}$ ) at which maximum isometric force was produced. Specific isometric force was calculated because muscles that differ in size, but are otherwise the same, produce the same specific force. Specific isometric force was calculated as force/CSA. Isometric force is the sum of that produced by all of the filaments in the muscle's cross section because the filaments are acting in parallel and their forces add. Because the filaments are arranged in a highly regular array, the number of filaments per unit CSA is a constant and thus CSA can be used as a measure of the number of filaments

\section{Statistical analysis}

All data are presented as mean values \pm standard error of the mean (SEM) (cohort size stated per experiment). Muscle fiber sizing was performed using SigmaScan Pro5 software (SPSS Inc., USA). All statistical analyses were performed using either the Student $t$-test or the chi-squared analysis using Sigmastat 3.1 software (Systat, USA).

\section{Results}

\section{Single intravenous administration of AAV8ProMyo leads to} a systemic increase in muscle mass

MF-1 male mice were weighed at weekly intervals from 2 weeks prior to the administration of the vector, thus ensuring mass-matched cohorts, until 10 weeks postadministration. Blood samples were recovered from control and treated cohorts to assess transgene expression by western blotting at 1,4 , and 10 weeks post administration. At a 4-week time point and at the end of the experiment, TA and gastrocnemius muscles were recovered and weighed.

Western blots to detect myostatin propeptide demonstrated expression in MF-1-treated mice at all time points analyzed (weeks 1-10), with no evidence that the level of expression altered with time (Fig. 1A). Using an anti-mouse $\operatorname{IgG}_{2 a}$ antibody to detect transgene expression, an extra band was noted on the western blot that localizes with $\mathrm{IgG}_{2 \mathrm{a}}$, and the fusion transgene product of ProMyo had a higher position on a western blot membrane. MF-1 male mice treated with AAV8ProMyo demonstrated a significant increase in gross mass by 4 weeks postinjection $(p=0.036)$, with the difference being maintained until the end of the experiment, week 10 ( $p=0.014$, Fig. 1B). There was a systemic increase in muscle mass from muscles recovered from AAV8ProMyo-treated mice, typified by increases in both the TA and gastrocnemius muscle by $24.8 \%(p<0.001)$ and $26.9 \%(p=0.010)$, at 10 weeks postadministration (Fig. $1 \mathrm{C}-\mathrm{D})$. 
A

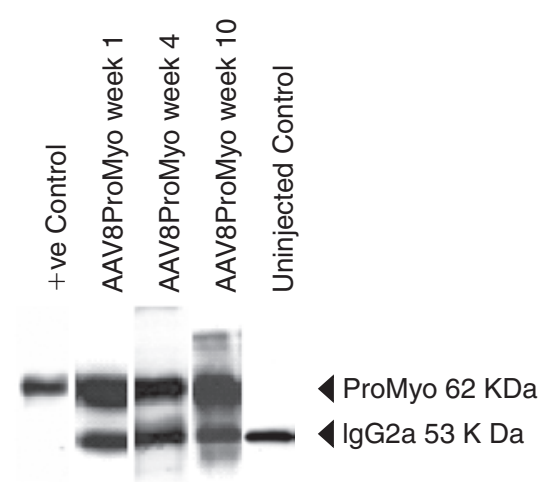

B

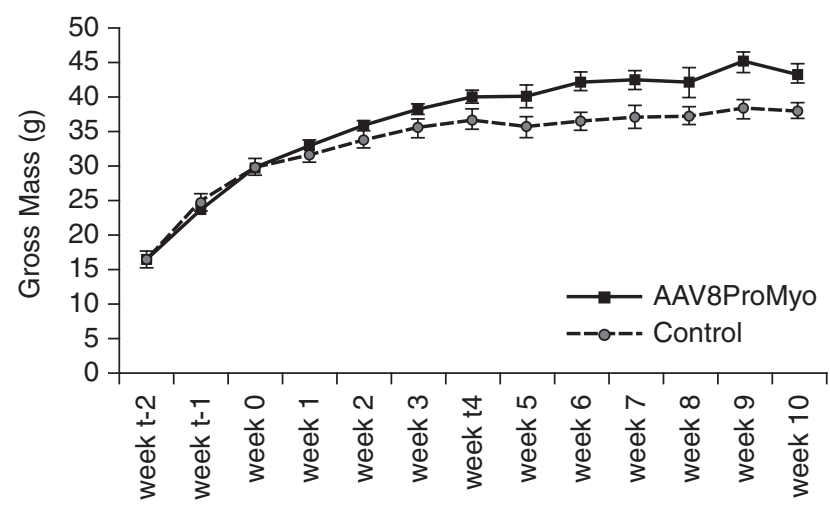

C

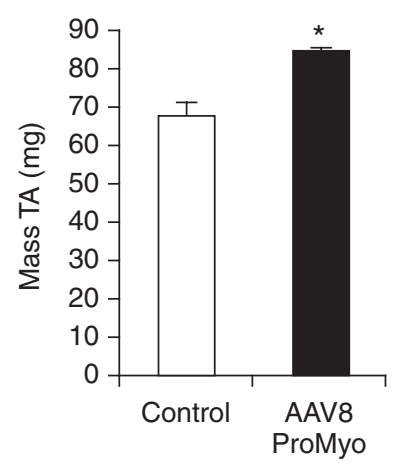

D

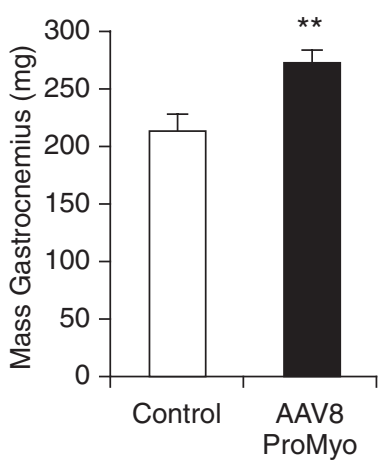

FIG. 1. AAV8-mediated transfer leads to sustained myostatin propeptide expression and increases in both gross body and individual muscle masses. (A) Western blot analysis of ProMyo transgene expression from pooled serum samples at 1, 4, and 10 weeks postinjection. (B) Gross body mass recorded at weekly intervals, starting from 2 weeks prior to gene transfer until 10 weeks postinjection (control $n=8$ weeks $1-4, n=4$ weeks $5-10$; AAV8ProMyo $n=10$, weeks $1-4$ and $n=5$ weeks 5-10). (C) Mass of TA and gastrocnemius muscles recovered at 10 weeks postinjection. Error bars are mean \pm SEM (control $n=4$; AAV8ProMyo $n=$ $\left.5,{ }^{*} p<0.001,{ }^{* *} p<0.01\right)$.

\section{Myostatin inhibition in adult muscle results in muscle fiber} hypertrophy and not hyperplasia

Next we examined individual muscle fibers to determine the cellular changes brought about by exposure to the myostatin propeptide. Given the systemic increase in muscle mass, it was important to assess whether the increase resulted from an increase in muscle hyperplasia, muscle hy- pertrophy, or both. Slow (soleus) and fast (EDL) muscles were recovered and examined histologically. First, the muscles were sectioned at 10 different intervals along the length of the muscle, allowing the maximal cross-sectional area (CSA) for both the soleus and EDL muscles to be determined; the subsequent analysis of individual fiber area was performed from tissue sections that generated the maximal CSA. Tissue sections were immunostained using an anti-laminin antibody to define the muscle fibers.

At the 10-week time point, the maximal CSA of the muscle was increased significantly in both soleus and EDL muscles recovered from AAV8ProMyo-treated mice: $31.7 \%(n=$ $4, p=0.004)$ and $51.7 \%(n=4, p=0.014)$, respectively (Fig. 2A). Analysis of CSA of individual muscle fibers for soleus and EDL yielded significant shifts from smaller fibers to larger fibers. The mean myofiber size for soleus increased from $1306.7 \mathrm{~mm}^{2}$ in controls to $1764.0 \mathrm{~mm}^{2}$ in AAV8ProMyo animals, with a general shift to larger fibers $\left(\chi^{2}=1099.31,18\right.$ df $p<0.001$ ). The mean myofiber size in EDL increased from $1115.5 \mathrm{~mm}^{2}$ in controls to $1513.1 \mathrm{~mm}^{2}$ in AAV8ProMyo animals, also demonstrating a general shift toward larger fibers $\left(\chi^{2}=625.10,18 \mathrm{df} p<0.001\right)$ (Fig. 2B).

\section{Satellite cell number and myonuclei number per fiber are unaffected by myostatin propeptide but the myofiber nuclear-to-cytoplasmic ratio is decreased}

EDL muscles were recovered at 8 weeks postinjection from control $(n=6)$ and AAV8ProMyo $(n=6)$ animals. The average number of satellite cells present on resting myofibers obtained from control or AAV8ProMyo EDL muscles was analyzed, and no significant difference was demonstrated in satellite cell number per myofiber $(7.27 \pm 0.28$ compared to $6.95 \pm 0.31$, respectively) (Fig. 3A).

The number of DAPI-positive myonuclei per myofiber from EDL muscles was quantified from the individual teased fibers, revealing no significant change between control and AAV8ProMyo-treated myofibers (361.1 \pm 7.5 compared to $370.6 \pm 7.6$, respectively) (Fig. $3 \mathrm{~B}$ ), suggesting that the myofiber nuclear content is not affected by myostatin propeptide administration.

MHC staining revealed that all fiber types showed a significant increase in CSA in the AAV8ProMyo-treated cohort (type I $495.5 \pm 25.2$ vs. $686.5 \pm 19.1(p<0.001)$, type IIa $530.8 \pm 9.8$ vs. $652.4 \pm 15.6(p<0.001)$ and type IIb $2581.7 \pm$ 39.0 vs. $3625.7 \pm 55.2(p<0.001)$; Fig. 3C). Importantly, however, when these data were expressed as a ratio of nuclei-to-cytoplasm (DAPI $/ \mu \mathrm{m}^{2}$ ), there was a significant reduction in the ratio for type I, IIa, and IIb myofibers from the AAV8ProMyo-treated cohort, when compared to control $(0.834 \pm 0.080$ vs. $0.555 \pm 0.168(p<0.001), 0.700 \pm 0.013$ vs. $0.584 \pm 0.014(p<0.001)$ and $0.154 \pm 0.003$ vs. $0.107 \pm 0.002$ $(p<0.001)$, respectively) (Fig. 3D).

\section{Physiological improvement in muscle force is restricted to slow muscle}

From a separate experiment, the physiological function of freshly recovered soleus and EDL muscles at a 4-week time point from euthanized animals, was tested, followed by measurement of muscle CSA. In this experiment, the increase in mass of soleus muscles recovered from the myostatin propeptide cohort was $14.2 \%(p=0.007)$, and this was reflected in an increase in CSA of $12.6 \%(p=0.006)$. The tetanic 


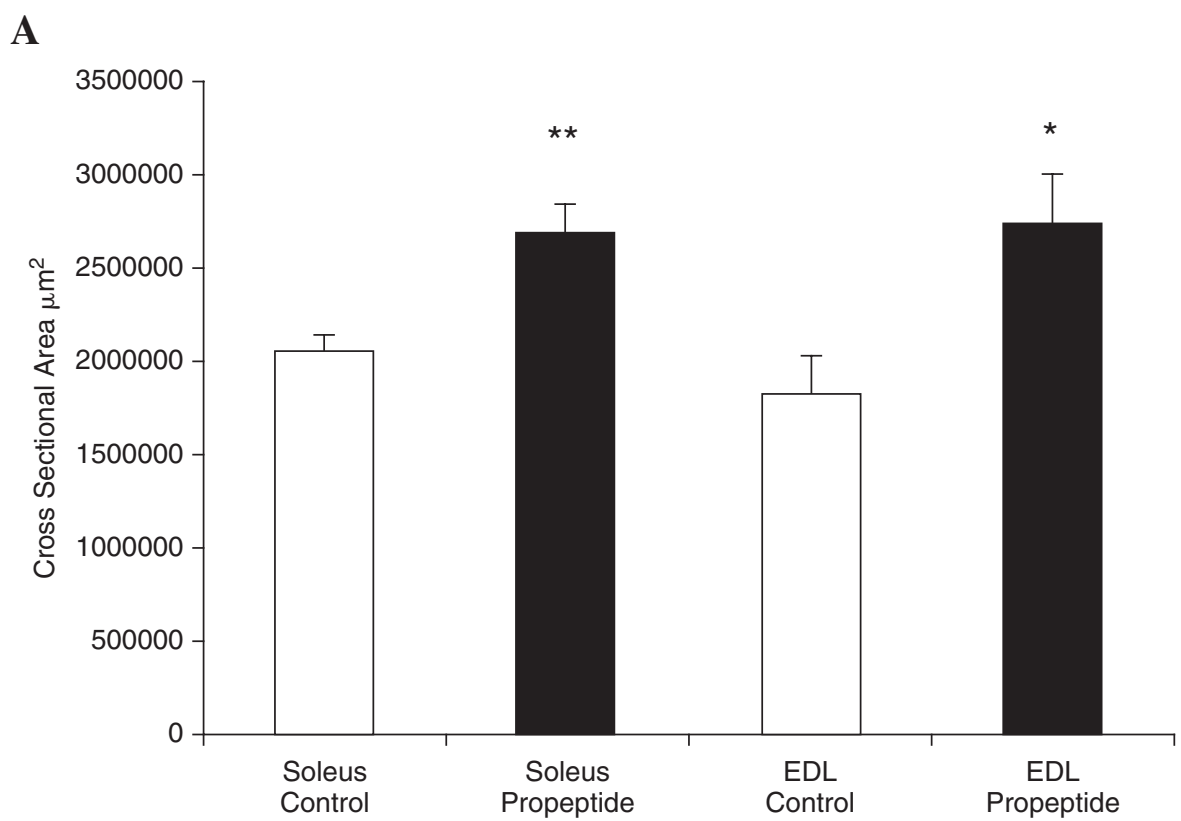

B
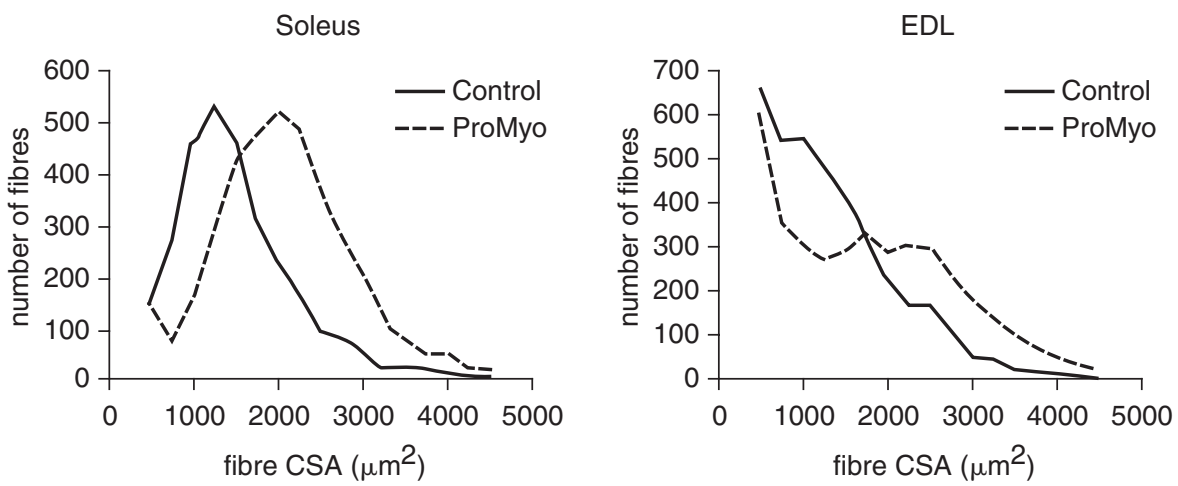

FIG. 2. Cross-sectional area of the entire muscle and individual muscle fibers is increased in both slow and fast muscle. Soleus and EDL muscles were recovered at 10 weeks postinjection (controls $n=4$, AAV8ProMyo $n=5$ ). (A) Maximal CSA of entire muscles. Error bars are mean \pm SEM $\left({ }^{* *} p<0.01,{ }^{* * *} p<0.05\right)$. (B) CSA analysis of individual muscle fibers on soleus and EDL muscle (chi-squared analysis performed on data sets, both soleus and EDL $p<0.001$ ).

isometric force generated from soleus muscles excised from the AAV8ProMyo cohort was significantly greater than that of the control (256.7 $\mathrm{mN}$ vs. $220.6 \mathrm{mN}$, respectively; $p=$ 0.008) (Fig. 4A). This increase in force of $16.0 \%$ was reflective of the increases in mass and CSA; however, when the specific force was calculated, which takes account of the CSA, the results between the control and AAV8ProMyo group were normalized ( $p=0.760$; Fig. $4 \mathrm{~B}$ ). EDL muscles recovered from the myostatin propeptide-treated group were heavier by $18.1 \%(p=0.005)$, with an increase in CSA of $15.8 \%(p=0.008)$. Neither the tetanic isometric force of the AAV8ProMyo EDL muscles nor their specific forces differed from those of the control; thus the force improvement was restricted to the slow muscle phenotype (Fig. 4C,D).

\section{Discussion}

This study has focused on the application of AAV8ProMyo-mediated systemic gene transfer to normal MF-1 mice to evaluate the histopathological and physiolog- ical effects in both slow and fast muscle. In vitro analysis of cultured C2C12 myoblasts with conditioned medium derived from HEK293T cells transfected with pProMyo demonstrated a significant increase in the proliferative capacity, compared to $\mathrm{C} 2 \mathrm{C} 12$ myoblasts grown in conditioned medium from sham-transfected HEK293T cells (data not shown). Subsequently, the in vivo effect of myostatin propeptide was assessed. AAV8ProMyo was administered by tail vein injection into 6-week-old MF-1 male mice, and their gross mass was recorded at weekly intervals for 10 weeks. Expression of gene transfer-mediated myostatin propeptide was evident in the serum of injected mice at the first time point analyzed (1 week), with no evidence that the amount of expression declined throughout the experiment. At 4 weeks postinjection, there was a significant difference in the gross mass of the treated cohort, which was maintained and became more significant up to the end of the experiment. At 4 and 10 weeks postinjection, the TA and gastrocnemius muscles were recovered and weighed. The mass of both the TA and gastrocnemius muscles was increased significantly 
A

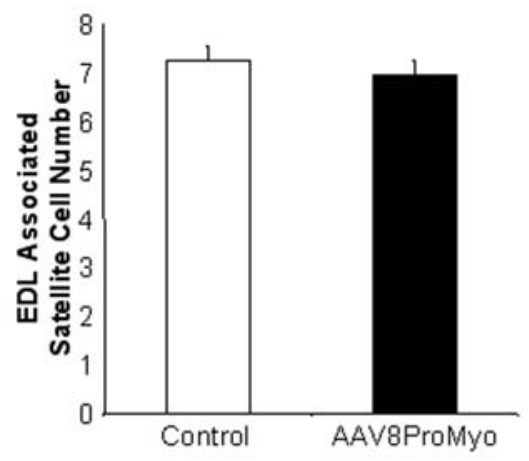

C

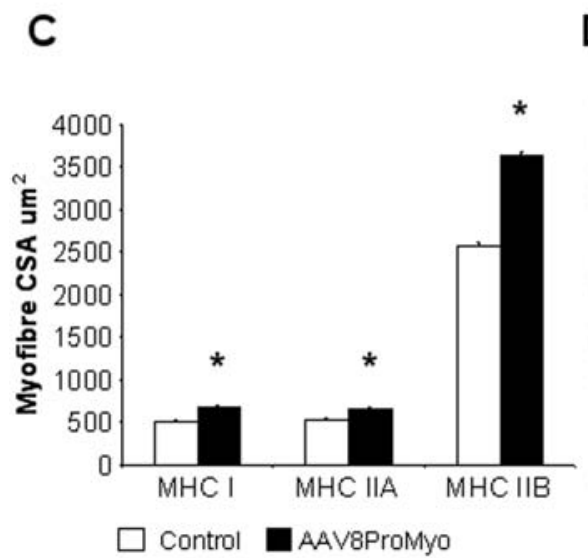

B

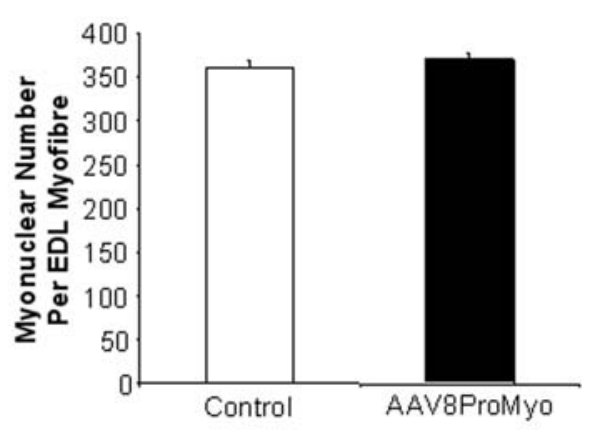

D

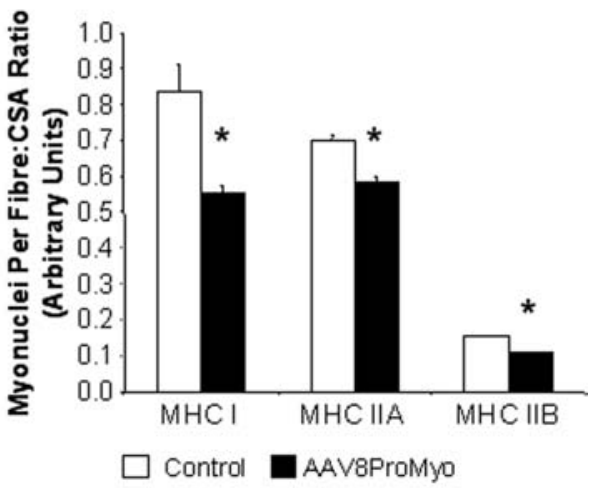

FIG. 3. Hypertrophy without hyperplasia is independent of satellite cell activity and results in a decrease in the nuclearto-cytoplasmic ratio. EDL muscles were recovered at 8 weeks postinjection (control $n=6$, AAV8ProMyo $n=6$ ). (A) Average satellite cell number per myofiber in control and AAV8ProMyo-treated EDL muscles. (B) Average myonuclei number per EDL myofiber. (C) Average CSA of individual type I, IIa, and IIb myofibers $\left({ }^{*} p<0.001\right)$. (D) Ratio of EDL myonuclei per myofiber-to-CSA ratio of individual type I, IIa, and IIb myofibers $\left({ }^{*} p<0.001\right)$.

in AAV8ProMyo-treated animals at 4 weeks (data not shown), with this degree of increase being maintained at the 10 -week time point. This increase in whole body mass and individual muscle mass is consistent with previous postnatal inhibition of myostatin in different strains of mice. ${ }^{35-38}$

Laminin- $\alpha 2$ is expressed in the basal lamina surrounding myofibers, and therefore was used to assess the gross CSA and CSA of individual fibers. Histological examination of midbelly sections of soleus and EDL muscle recovered at 10 weeks after administration of AAV8ProMyo demonstrated that there was a significant increase in CSA area of both muscles. Individual muscle fibers for soleus and EDL yielded significant shifts from smaller fibers to larger fibers; the mean myofiber size for both muscles increased significantly, showing a general shift to larger fibers, consistent with previous findings. ${ }^{35,37,38}$ The increase in gross CSA was greater for the EDL than the soleus muscle, but of the same magnitude to that described in myostatin null mice. ${ }^{39}$ However, it is important that there was no increase in the total number of myofibers within each muscle in this experiment, nor was there an increase in the number of myonuclei per CSA myofiber, thus confirming that the postnatal increase in muscle mass results from muscle hypertrophy and not hyperplasia. The differential in the response between soleus and EDL muscles may be due in part to a greater amount of ActIIb receptors being expressed on the surface of EDL muscle, ${ }^{39}$ or because the intrinsic level of myostatin is greater in 'fast' myosin type IIb myofibers, ${ }^{40}$ which could potentially lead to a greater release from the inhibitory effects of myostatin on the EDL muscle. The general increase in fiber CSA in both the soleus and EDL muscles is indicative of a general shift in fiber type from an oxidative to a glycolytic phenotype. This is supported by the downregulation of muscle-specific genes such as slow myosins in myostatin null mice. ${ }^{41,42}$

Given the histological results suggesting that the increase in adult muscle mass was solely due to muscle hypertrophy, we wished to study the in vivo effects of myostatin inhibition on satellite cells. Adult satellite cells that associate with myofibers are a model of self-renewal of muscle; their activation essentially controls the number of myoblasts and the number of myoblasts that terminally differentiate and fuse into postmitotic muscle fibers. ${ }^{11,43,44}$ Therefore, assessing the proliferative capacity of myofiber-associated satellite cells is a model of muscle regeneration. In a separate experiment, EDL muscles were recovered at 8 weeks postadministration of AAV8ProMyo with single myofibers being isolated and satellite cell analyses made. There was no significant difference in the number of satellite cells associated with myofibers from EDL muscles of control and myostatin propeptide-treated mice. Given that we provide no evidence for a greater number of myonuclei per myofiber, but an increase in CSA, this demonstrates that the nuclear-to-cytoplasmic ra- 
A

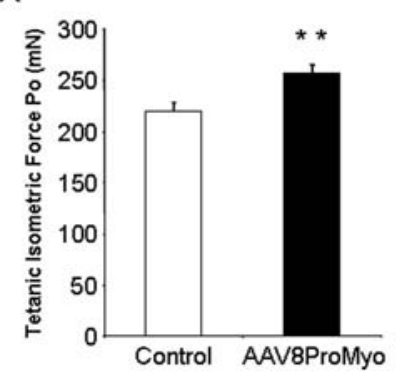

C

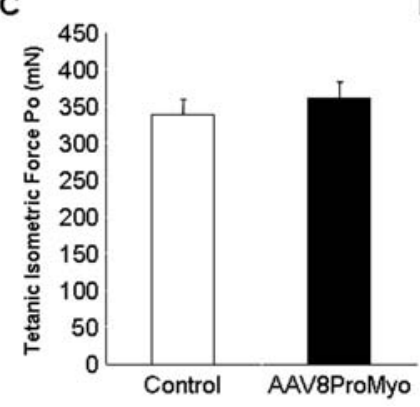

B

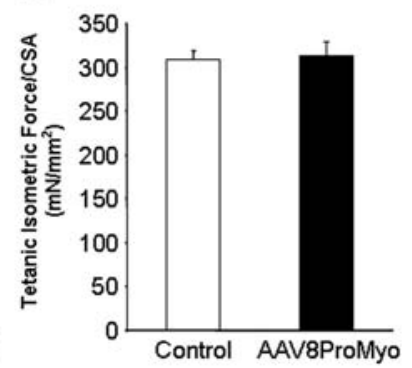

D

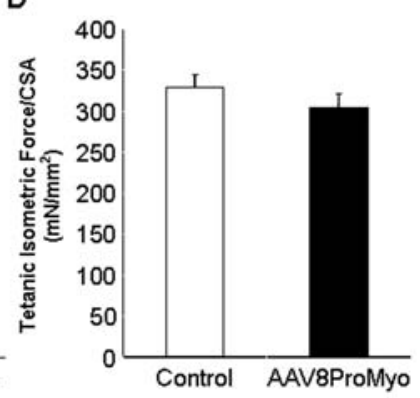

FIG. 4. Intravenous administration of myostatin propeptide leads to greater improvements in functional properties of soleus but not EDL muscles. Soleus and EDL muscles were recovered at 4 weeks postinjection $(n=7)$. Maximal tetanic isometric force was determined for soleus (A) and EDL (C) muscles. Maximal tetanic isometric force was normalized against the CSA to generate the specific force for soleus (B) and EDL (D) muscles. Error bars shown as mean \pm SEM $(* * p<0.01)$.

tio is lower in both slow and fast muscles and is not dependent on myosin fiber type. This observation seems to be at odds from the data that can be inferred from observations in myostatin null mice, in which the extent of the increase in CSA mainly reflected the increase in myofiber number. ${ }^{39}$ Indeed, from our data, the increase in CSA was greater in MHC type IIb fibers than type I or type IIa, again suggesting that this increased response in type IIb fibers could be due to a greater amount of ActIIb receptors being expressed on the surface of type IIb fibers. ${ }^{40}$

Our data are supported by other observations following postnatal inhibition of myostatin in which an increase in muscle mass solely resulted from muscle hypertrophy. ${ }^{38}$ Taken together, these observations suggest that the hypertrophy resulting from postnatal inactivation of myostatin does not affect the recruitment of muscle precursor cells to the fiber, which is contrary to myostatin null mice in which there is a positive correlation with satellite cell activation and myoblast recruitment. ${ }^{45,46}$ Furthermore, this positive effect in null mice may be restricted to the promotion of secondary myogenesis, which is thought to promote a fast myosin type IIb phenotype. ${ }^{47}$ Overall, this suggests that postnatal inactivation of myostatin does not lead to enhanced activation of satellite cells, which contradicts the current dogma of myostatin inhibition being implicit in satellite cell activation. Whether or not the lack of maintenance of a defined nuclear-

to-cytoplasmic ratio in postnatal inactivation of myostatin impairs normal cellular function may have implications for its use in the treatment of muscle wasting disorders requires further investigation, especially given the general glycolytic shift and dysregulation of mitochondrial number and structure noted in fast muscle. ${ }^{48}$

We next examined the effects on force generation for both soleus and EDL muscles using an ex vivo protocol. Muscles were recovered at 4 weeks post AAV8ProMyo injection, with both soleus and EDL muscles having a significant increase in muscle mass. The tetanic isometric force significantly increased in soleus muscle in the AAV8ProMyo group and was consistent with the increase in muscle mass. When normalized against the CSA, thus generating the specific force, we found that there was no difference between treatment groups. However, an increase in mass of EDL muscle was not accompanied with a concomitant increase in tetanic isometric force; thus, when normalized against CSA, there was a trend that the specific force was lower, but not significantly so. Therefore, there is a disparity in the functional response to myostatin inhibition between adult soleus and EDL muscles. The enhancement of function in outbred mice muscle following myostatin inhibition is restricted to a slow muscle phenotype. Similar results have been reported on a C57Bl/10 background following postnatal inhibition of myostatin. Although there was a general increase in muscle mass and CSA, interestingly there was no functional improvement when another fast muscle, TA, was analyzed. ${ }^{38}$

In myostatin null mice, the tetanic isometric forces of soleus and EDL muscles are not increased but the specific force of EDL is reduced; the corollary of this is that the EDL is more prone to contraction-induced injury, ${ }^{39}$ consistent with our findings in adult muscle. This suggests that for fast muscle phenotypes there may be a threshold above which an additional increase in muscle mass is deleterious to muscle function by breaching a cytoplasmic-to-nuclear ratio. Indeed, in mice heterozygous for myostatin mutation, the increase in muscle mass is moderate compared to knockout mice, but significant compared to controls. However, the higher tetanic isometric force does not compromise the specific force, ${ }^{39}$ which is supported by the heterozygous advantage noted in both dogs and humans. ${ }^{49,50}$ Additionally, there is a difference in the fiber length/muscle length ratio for soleus (0.7) and EDL (0.44) muscles, which may also contribute to our findings. Potentially the degree of pennation is not so pronounced for the soleus muscle, whereas the pennation angle may surpass a threshold limit for which EDL function begins to be compromised.

Allied with the significant reduction in the specific force of the EDL muscle of myostatin null mice, the mitochondrial content is also lower ${ }^{39,48}$; interestingly, a reduction in force output is a clinical feature consistent with patients with a mitochondrial depletion disorder. ${ }^{51}$ It is seen that mitochondria serve as a fast $\mathrm{Ca}^{2+}$ sink, as well as generating adenosine triphosphate (ATP) for the active transport of cytosolic $\mathrm{Ca}^{2+}$ into the sarcoplasmic reticulum. ${ }^{52}$ Therefore, the result of the reduction in the oxidative capacity of the muscle cell may be that $\mathrm{Ca}^{2+}$ homeostasis is impaired, thereby perturbing the excitation-coupling reaction affecting force output. This theory is potentially supported by the fact that myostatin-deficient muscle has a reduced capillary density. ${ }^{53}$ The fact that the specific force of soleus muscle is not impaired in myostatin 
null mice suggests that, although there is a shift from an oxidative to a glycolytic phenotype, a reduction in the oxidative capacity may not be sufficient to reach a threshold below which an impairment of mitochondrial and $\mathrm{Ca}^{2+}$ homeostasis occurs, and thus the force output is unaffected. However, the effects on muscle cell mitochondrial content and $\mathrm{Ca}^{2+}$ homeostasis need to be evaluated in a model of postnatal myostatin inhibition to assess the feasibility of a myostatin inhibitory regimen in the management of muscle wasting disease in combination with exercise induced fatigue.

\section{References}

1. Thomas DR. Loss of skeletal muscle mass in aging: examining the relationship of starvation, sarcopenia and cachexia. Clin Nutr 2007;26:389-399.

2. Buckingham $\mathrm{M}$ et al. The formation of skeletal muscle: from somite to limb. J Anat 2003;202:59-68.

3. McPherron AC, Lawler AM, Lee SJ. Regulation of skeletal muscle mass in mice by a new TGF-beta superfamily member. Nature (1997:387:83-90.

4. Lee SJ, McPherron AC. Regulation of myostatin activity and muscle growth. Proc Natl Acad Sci USA 2001;98:9306-9311.

5. McPherron AC, Lee SJ. Double muscling in cattle due to mutations in the myostatin gene. Proc Natl Acad Sci USA 1997; 94:12457-12461.

6. Grobet L, et al. A deletion in the bovine myostatin gene causes the double-muscled phenotype in cattle. Nat Genet 1997;17:71-74.

7. Schuelke M, et al. Myostatin mutation associated with gross muscle hypertrophy in a child. N Engl J Med 2004;350:26822688.

8. Clop A, et al. A mutation creating a potential illegitimate microRNA target site in the myostatin gene affects muscularity in sheep. Nat Genet 2006;38:813-818.

9. Shelton GD, Engvall E. Gross muscle hypertrophy in whippet dogs is caused by a mutation in the myostatin gene. Neuromuscul Disord 2007;17:721-722.

10. Gonzalez-Cadavid NF, et al. Organization of the human myostatin gene and expression in healthy men and HIV-infected men with muscle wasting. Proc Natl Acad Sci USA 1998;95:14938-14943.

11. Zammit PS, et al. Kinetics of myoblast proliferation show that resident satellite cells are competent to fully regenerate skeletal muscle fibers. Exp Cell Res 2002;281:39-49.

12. Yarasheski KE, Bhasin S, Sinha-Hikim I, Pak-Loduca J, Gonzalez-Cadavid NF. Serum myostatin-immunoreactive protein is increased in 60-92 year old women and men with muscle wasting. J Nutr Health Aging 2002;6:343-348.

13. Amthor $\mathrm{H}$, et al. The regulation and action of myostatin as a negative regulator of muscle development during avian embryogenesis. Dev Biol 2002;251:241-257.

14. Hill JJ, et al. The myostatin propeptide and the follistatinrelated gene are inhibitory binding proteins of myostatin in normal serum. J Biol Chem 2002;277:40735-40741.

15. Thies RS, et al. GDF- 8 propeptide binds to GDF- 8 and antagonizes biological activity by inhibiting GDF-8 receptor binding. Growth Factors 2001;18:251-259.

16. Zimmers TA, et al. Induction of cachexia in mice by systemically administered myostatin. Science 2002;296:1486-1488.

17. Hill JJ, et al. Regulation of myostatin in vivo by growth and differentiation factor-associated serum protein-1: a novel protein with protease inhibitor and follistatin domains. Mol Endocrinol 2003;17:1144-1154.
18. Wolfman, NM, et al. (2003). Activation of latent myostatin by the BMP-1/tolloid family of metalloproteinases. Proc Natl Acad Sci U S A 100: 15842-15846.

19. Thomas M, et al. Myostatin, a negative regulator of muscle growth, functions by inhibiting myoblast proliferation. J Biol Chem 2000;275:40235-40243.

20. Langley B, Thomas M, Bishop A, Sharma M, Gilmour S, Kambadur R. Myostatin inhibits myoblast differentiation by down-regulating MyoD expression. J Biol Chem 2002;277: 49831-49840.

21. Rebbapragada A, Benchabane H, Wrana JL, Celeste AJ, Attisano L. Myostatin signals through a transforming growth factor beta-like signaling pathway to block adipogenesis. Mol Cell Biol 2003;23:7230-7242.

22. Amthor H, Otto A, Macharia R, McKinnell I, Patel K. Myostatin imposes reversible quiescence on embryonic muscle precursors. Dev Dyn 2006;235:672-680.

23. Yang W, Zhang Y, Li Y, Wu Z, Zhu D. Myostatin induces cyclin D1 degradation to cause cell cycle arrest through a phosphatidylinositol 3-kinase/AKT/GSK-3 beta pathway and is antagonized by insulin-like growth factor 1 . J Biol Chem 2007;282:3799-3808.

24. von Wichert G, et al. Regulation of cyclin D1 expression by autocrine IGF-I in human BON neuroendocrine tumour cells. Oncogene 2005;24:1284-1289.

25. McCroskery S, Thomas M, Maxwell L, Sharma M, Kambadur R. Myostatin negatively regulates satellite cell activation and self-renewal. J Cell Biol 2003;162:1135-1147.

26. Philip B, Lu Z, Gao Y. Regulation of GDF-8 signaling by the p38 MAPK. Cell Signal 2005;17:365-375.

27. Guo W, Flanagan J, Jasuja R, Kirkland JL, Jiang L, Bhasin S. The effects of myostatin on adipogenic differentiation of human bone marrow-derived mesenchymal stem cells are mediated through cross-communication between smad3 and WNT/beta-catenin signaling pathways. J Biol Chem 2008; 283:9136-9145.

28. Warner DR, Greene RM, Pisano MM. Interactionbetween Smad 3 and Dishevelled in murine embryonic craniofacial mesenchymal cells. Orthod Craniofac Res 2005;8:123-130.

29. Lee SJ, et al. Regulation of muscle growth by multiple ligands signaling through activin type II receptors. Proc Natl Acad Sci USA 2005;102:18117-18122.

30. Lee SJ. Quadrupling muscle mass in mice by targeting TGFbeta signaling pathways. PLoS ONE 2007;2:e789.

31. Nakai H, Fuess S, Storm TA, Muramatsu S, Nara Y, Kay MA. Unrestricted hepatocyte transduction with adeno-associated virus serotype 8 vectors in mice. J Virol 2005;79:214-224.

32. Yue Y, Dongsheng D. Development of multiple cloning site cis-vectors for recombinant adeno-associated virus production. Biotechniques 2002;33:672,674,676-678.

33. Otto A, et al. Canonical Wnt signalling induces satellite-cell proliferation during adult skeletal muscle regeneration. J Cell Sci 2008;121:2939-2950.

34. Curtin NA, Woledge RC. Power and efficiency: how to get the most out of striated muscle. Adv Exp Med Biol 1993;332: 729-733; discussion 733-724.

35. Bogdanovich S, Perkins KJ, Krag TO, Whittemore LA, Khurana TS. Myostatin propeptide-mediated amelioration of dystrophic pathophysiology. FASEB J 2005;19:543-549.

36. Bogdanovich $S$, et al. Functional improvement of dystrophic muscle by myostatin blockade. Nature 2002;420:418-421.

37. Bartoli M, et al. AAV-mediated delivery of a mutated myostatin propeptide ameliorates calpain 3 but not alpha-sarcoglycan deficiency. Gene Ther 2007;14:733-740.

38. Qiao C, et al. Myostatin propeptide gene delivery by adeno- 
associated virus serotype 8 vectors enhances muscle growth and ameliorates dystrophic phenotypes in mdx mice. Hum Gene Ther 2008;19:241-254.

39. Mendias CL, Marcin JE, Calerdon DR, Faulkner JA. Contractile properties of EDL and soleus muscles of myostatindeficient mice. J Appl Physiol 2006;101:898-905.

40. Carlson CJ, Booth FW, Gordon SE. Skeletal muscle myostatin mRNA expression is fiber-type specific and increases during hindlimb unloading. Am J Physiol 1999);277:R601-R606.

41. Girgenrath S, Song K, Whittemore LA. Loss of myostatin expression alters fiber-type distribution and expression of myosin heavy chain isoforms in slow- and fast-type skeletal muscle. Muscle Nerve 2005;31:34-40.

42. Steelman CA, Recknor JC, Nettleton D, Reecy JM. Transcriptional profiling of myostatin-knockout mice implicates Wnt signaling in postnatal skeletal muscle growth and hypertrophy. FASEB J 2006;20:580-582.

43. Collins CA, et al. Stem cell function, self-renewal, and behavioral heterogeneity of cells from the adult muscle satellite cell niche. Cell 2005;122:289-301.

44. Heslop L, Morgan JE, Partridge TA. Evidence for a myogenic stem cell that is exhausted in dystrophic muscle. J Cell Sci 2000;113(Pt 12):2299-2308.

45. McCroskery S, et al. Improved muscle healing through enhanced regeneration and reduced fibrosis in myostatin-null mice. J Cell Sci 2005;118:3531-3541.

46. Wagner KR, Liu X, Chang X, Allen RE. Muscle regeneration in the prolonged absence of myostatin. Proc Natl Acad Sci USA 2005;102:2519-2524.

47. Whittemore LA, et al. Inhibition of myostatin in adult mice increases skeletal muscle mass and strength. Biochem Biophys Res Commun 2003;300:965-971.
48. Amthor $\mathrm{H}$, et al. Lack of myostatin results in excessive muscle growth but impaired force generation. Proc Natl Acad Sci USA 2007;104:1835-1840.

49. Mosher DS, et al. A mutation in the myostatin gene increases muscle mass and enhances racing performance in heterozygote dogs. PLoS Genet 2007;3:e79.

50. Kostek MA, et al. The Myostatin A2379G Polymorphism and Muscle Strength Among African Americans: 1721: Board \#9 May 31 8:00 AM 9:30 AM. Med Sci Sports Exerc 2007;39:S280.

51. Mancuso M, et al. Mitochondrial DNA depletion: mutations in thymidine kinase gene with myopathy and SMA. Neurology 2002;59:1197-1202.

52. Andrade FH, McMullen CA, Rumbaut RE. Mitochondria are fast $\mathrm{Ca} 2+$ sinks in rat extraocular muscles: a novel regulatory influence on contractile function and metabolism. Invest Ophthalmol Vis Sci 2005;46:4541-4547.

53. Rehfeldt C, et al. Effects of the compact mutant myostatin allele Mstn (Cmpt-dl1Abc) introgressed into a high growth mouse line on skeletal muscle cellularity. J Muscle Res Cell Motil 2005;26:103-112.

Address reprint requests to: George Dickson

School of Biological Sciences Royal Holloway-University of London Egham, Surrey, TW20 0EX, United Kingdom

Received: November 7, 2008 Accepted: December 7, 2008

E-mail: g.dickson@rhul.ac.uk 
